\title{
28 Research Square \\ Genetic and Clinical Phenotypic Analysis of Familial Stapes Sclerosis Caused by a NOG Mutation
}

\section{Rong Yu}

First Affiliated Hospital of Nanchang University https://orcid.org/0000-0002-1689-0226

\section{HONGQUN JIANG}

First Affiliated Hospital of Nanchang University

WU GEN LUO ( $\sim$ luowugen@126.com )

First Affiliated Hospital of Nanchang University

\section{Research article}

Keywords: gene, deafness, stapes sclerosis

Posted Date: August 13th, 2020

DOI: https://doi.org/10.21203/rs.3.rs-42135/v1

License: (1) This work is licensed under a Creative Commons Attribution 4.0 International License.

Read Full License 


\section{Abstract}

Background $\mathbb{X}$ Noggin protein encoded by NOG gene can interfere with the binding of bone morphogenetic protein to its receptor, thus affecting bone and joint develop pment. The symptoms include abnormal skeletal development and conductive deaf ness

Methods: In a Retrospective study, clinical data of the proband and her family members include 8 people and 50 healthy normal controls were collected. Secon d-generation sequencing were performed on peripheral blood samples from them.

Results囚The sequencing analysis indicated that, in the probrand, the NOG gene included c.532T>C (cytosine deletion), leading to an amino acid change. The prob and's father, grandmother, second sister, and third sister also had this mutation, whereas those with normal phenotypes did not have the mutation.

Conclusion $\triangle A$ Analysis of this family showed that the new presentation of c.532T $>C$ mutation in the NOG gene resulted in syndrome-type autosomal dominant inheritan ce reflected in a mild clinical phenotype, which is of great significance for further studies of the clinical phenotype and pathogenesis of stapetectopia.

\section{Background}

Conductive deafness is a type of hearing loss caused by lesions in the outer and middle ear. The passage of external sound waves into the inner ear is obstructed by pathological factors affecting sound transmission system in the ear. Congenital causes are common malformation of the outer ear, hypoplasia of the tympanum, malform ation of ossicular chain, familial otosclerosis, etc. Acquired causes are commonly seen in external auditory canal obstruction by cerumen, foreign bodies, inflammation, scars, tumors, etc., or by tympanic membrane trauma, perforation, thickening and adhesion, middle ear effusion or pus, cholesteatoma, fracture of the auditory chain, ear sclerosis, tumor, etc. Stapes ankylosis is rare but can cause early conductive deafness.[1-4]This is difficult to differentiate from a common etiology of conductive deafness, otosclerosis, and is often overlooked, delaying treatment. Stapes ankylosis may be associated with skeletal dysplasia, such as osteogenesis imperfecta type I (MIM 166200).[5-7]Skeletal dysplasia is a common congenital malformation and a common phenotype in many inherited disorders.

Abnormal bone and joint development are the most common congenital malform ations. Mutations of the NOG gene (OMIM602991) on chromosome 17q21-q22 can lead to multiple autosomal dominant genetic diseases. The noggin protein encoded by the NOG gene can interfere with the binding of bone morphogenetic protein to its receptor, thus affecting bone and joint development, which may be manifested as skeletal dysplasia and conductive deafness[8-11]. Skeletal dysplasia includes proximal interphalangeal joint fusion syndrome type A (OMIM 185800), multiple osseous junction syndrome type 1 OMIM 186500, tarsal and carpal joint fusion syndrome (OMIM 186570), short finger syndrome type B2 (OMIM 611377), and stapes stiffness with a wide thumb and toes (OMIM 184460)(8). To improve the 
understanding of this rare disease, NOG gene analysis was performed in a family with stapes ankylosis in China

Few studies have investigated the mechanism and correlates of stapes ankylosis in China, and a gold standard for pathological diagnosis is still lacking. Clinical diagnosis is mainly based on family history, genetic history, genetic testing, and phenotypic signs. Currently, surgery is the only specific and effective treatment. Therefore, a thorough understanding of the disease is important. In this study, we performed a clinical examination and genetic mutation analysis of a Han Chinese family with a suspected clinical manifestation of stapes ankylosis.

\section{Methods}

Members of the family in this study were from Jiangxi Province, China. The ten-member family, two of whom had died, included three generations. A detailed medical history was taken of the eight living family members, and all suspected patients underwent a detailed physical examination, including a specialist examination of the ear, height measurement, hand X-ray, and hearing tests. Other family members were identified as patients through detailed questioning. Peripheral venous blood was collected from the proband and other family members. The control group included venous blood samples from 50 normal controls in the molecular genetic database of our hospital. All adult subjects signed informed consent forms.

The proband and her three sisters, father, mother, grandmother, and uncle were screened for testing. Peripheral venous blood samples were extracted from the subjects with 3-5 mL of EDTA anticoagulant, and DNA was extracted using the Qiagen Genomic DNA Extraction Kit (Qiagen, Inc., Hilden, Germany) following the manufacturer's instructions. The DNA was then quantified and purity tested using a NanoDrop 1000 (Thermo Fisher Scientific, Waltham, MA, USA) and then stored at $-80^{\circ} \mathrm{C}$. After the extracted DNA was purified and tested for purity, it was sent to the company's laboratory for relevant genetic testing. The target genes of the samples were captured by liquid-phase capture technology, and high-throughput sequencing was performed using an Illumina HiSeq 2000 (Illumina, San Diego, CA, USA) at an average depth of $>200 X$.

The sequencing results were compared to the Human Gene Mutation Database (HG19), Locus Specific Mutation Database (HTTP://www.hgvs.org/dblist/glsdb.htm), dbSNP (v144), US National Center for Bio informatics, SPIDEX Database, and other databases. The Netgene2 Server was used to analyze and detect the pathogenicity of splicing mutations and other potentially pathogenic sites.

\section{Results}

Members of the family in this study were from Jiangxi Province, China. The ten-member family, two of whom had died, included three generations. A detailed medical history was taken of the eight living family members, and all suspected patients underwent a detailed physical examination, including a 
specialist examination of the ear, height measurement, hand X-ray, and hearing tests. Other family members were identified as patients through detailed questioning. Peripheral venous blood was collected from the proband and other family members. The control group included venous blood samples from 50 normal controls in the molecular genetic database of our hospital. All adult subjects signed informed consent forms. The proband was a 27-year-old woman of Han nationality from Jiangxi Province. The proband's parents denied inbreeding. The proband reported 4 years of progressive hearing loss with no history of otitis media, ototoxic drug use, or noise exposure. The proband was $168 \mathrm{~cm}$ in height and hyperopic, broad thumbs and toes, difficulty in bending thumbs, limited neck range of motion. Eardrums on both sides were intact. The Rinne and $512 \mathrm{~Hz}$ tes ts using a tuning fork were negative, the Weber test was biased to the side with sever e deafness, the Schwabach test showed bone conduction extension, and the Gelle test was negative, suggesting stapes plate fixation. Pure-tone audiometry indicated bilate ral conductive deafness. No obvious abnormalities were found in acoustic conductiv ity tests or on ear computed tomography (Fig. 1). The clinical diagnosis was stapes ankylosis.

The proband and her three sisters, father, mother, grandmother, and uncle were screened for testing. Peripheral venous blood samples were extracted from the subje cts with 3-5 mL of EDTA anticoagulant, and DNA was extracted using the Qiagen Genomic DNA Extraction Kit (Qiagen, Inc., Hilden, Germany) following the manufac turer's instructions. The DNA was then quantified and purity tested using a NanoDrop 1000 (Thermo Fisher Scientific, Waltham, MA, USA) and then stored at $-80^{\circ} \mathrm{C}$. After the extracted DNA was purified and tested for purity, it was sent to the company's laboratory for relevant genetic testing. The target genes of the samples were captured by liquid-phase capture technology, and high-throughput sequencing was performed using an Illumina HiSeq 2000 (Illumina, San Diego, CA, USA) at an average depth of $>200 X$.

Six family members had conductive hearing loss, stapes rigidity, hyperopia, and other phenotypic traits such as broad thumbs and/or toes, difficulty bending thumbs, decreased extremity and neck ROM (range of motion). The proband had conductive hearing loss, hyperopia, broad thumbs and difficulty bending thumbs, decreased extremity and neck ROM. The proband's first sister had no obvious problems. The proband's second sister had the same symptom with the probrand except difficulty bending thumbs. The probrand's third sister had the same symptom with the probrand except hyperopia. The proband's father showed the same conditions as the proband, and her mother was a congenitally deaf and mute. One of the proband's uncles, who had died in an accident, had hyperopia, conductive deafness. Another uncle was normal. The proband's grandmother had the same symptom with the probrand except decreased extremity and neck ROM (range of motion). The heights of the patients in the family were tall except the proband's grandmother, and their walking function was normal. There was no other family history. Autosomal dominant genetic characteristic cs noted in this family are shown in (Fig. 2).

The sequencing results were compared to the Human Gene Mutation Database (HG19), Locus Specific Mutation Database (HTTP: / / www.hgvs.org/ dblist/glsd b.htm), dbSNP (v144), US National Center for Bio informatics, SPIDEX Database, and other databases. The Netgene2 Server was used to analyze and detect the path ogennicity of splicing mutations and other potentially pathogenic sites. The DNA 
sequencing analysis indicated that the NOG heterozygous mutation, namely c.532t>C (code no. 532 nucleotide variation for cytosine by thymine), was present in the probrand' father and grandmother. The two sisters also carried the mutation. To verify that this was the pathogenic mutation that caused stapes antecosis, we screened hearing in 50 normal individuals from the Chinese populat ion for NOG gene mutations. None of the 50 had the mutation. Therefore, the c.532 T>C mutation does not belong to the polymorphic site and is considered pathogenic (Fig. 3).

We searched the Chinese literature and PubMed database to find relevant litera ture on the NOG gene mutation in a Chinese population. The results showed that nearly 40 mutations in the NOG gene have been identified, most of which are misse nse mutations located in the evolutionarily conserved region. Within this region is a functionally critical domain, suggesting a correlation between the region and the NOG protein. The c.532T>C mutation identified in this family has not been previously rep orted. The only clinical symptoms that were similar to those reported in our pedigree were brown et al. 2 . showed anaphylaxis stapes accompanied by broad thumb NOG was found to be absent in patients with fingers, hyperopia and syndactyly. In addition, the clinical characteristics of the affected individuals in this lineage are highly hetero geneous.

\section{Discussion}

Congenital stapes ankylosis syndrome is rare, the only thing consistent with this family's performance is David J. Brown once reported on an Italian family[2]. But it can cause early-onset conductive hearing loss. Congenital stapes ankylosis syndrome is difficult to distinguish from otosclerosis, the most common cause of conductive dea fness, and is therefore prone to delayed diagnosis. The skeletal abnormalities it causes are mild, making the syndrome harder to recognize. Mutations in the NOG gene can lead to stapes ankylosis, conductive hearing loss, and joint damage. In this study, we report a familial stapes stiffness syndrome in one family with conductive hearing loss. Further genetic testing in this study revealed that the patient and the affected family members all carried the same NOG gene mutation. The clinical diagnosis was stapes ankylosis syndrome, and all patients in the family had conductive deafness. All of the patients were hyperopic except one. Some of them have an inability to bend thumbs, and broad thumb and/or toes. Some of them have decreased extremity and neck ROM. The overall clinical phenotype of the patients in this family was relatively mild.

The c.532T $>C$ mutation in this family has not been reported before, but its clinical symptoms are similar to other NOG mutations.[12-14] .But they did not report the ophthalmological findings in detail. The key features that differentiate SYM1 and SYNS1 from stapes ankylosis with broad thumbs and toes and hyperopia include a characteristic physiognomy, hyperopia, and the absence of cervical vertebral fusion and symphalangism.

The reported results for NOG mutation sites and clinical phenotypes show that the diversity of NOG is reflected in the diversity of mutation sites, the diversity of clinical manifestations, and the diversity of disease courses and prognoses. Ideally, genotype - phenotype relationship analysis is performed on a 
large sample of patients, but due to the diversity of gene mutations, patients with identical mutations are limited in number. Therefore, it is difficult to determine the relationship between the mutations and clinical phenotype. Bayat et al. proposed a new mutation in NOG in which cysteine 230 was replaced in a Danish family, but none of the affected family members suffered hearing loss[15]. Another study reported that mutations in cysteine 184 caused no indication of hearing impairment. In the family reported here, both cysteine 184 and 230 were affected, resulting in stapes rigidity, hearing loss, and phalangeal dysfunction[16]. These findings confirm that mutations with the same NOG coding sequence can lead to different phenotypes and inter-family variation[17-25]. This suggests that disease expression can be independent of the location and type of NOG mutation.NOG Mutation contains a variety of syndromes.

The reported effects of otological surgery for stapes fixation caused by NOG protein mutation vary[2630]. In this study, surgical treatment was adopted in five ears (one patients), and hearing continued to improve after the operations. The follow-up period was 6 months.Patient's hearing improved by about 20 $\mathrm{dB}$ after surgery.Some reports describe progressive hearing loss due to bone regrowth 2-5years after surgery. In this study, the number of patients was small, and it was difficult to determine statistical significance or make treatment recommendations. The outcome of stapedectomy may also depend on genetic variation. Our current results were all based on the pure tone audiogram; the early audiogram did not assess the speech reception threshold. This threshold is also an important parameter and should be considered in the evaluation of surgical results.

\section{Conclusion}

Conductive hearing loss is most often caused by otitis media or other middle ear lesions. NOG mutations can lead to early-onset conductive hearing loss. In combina tion with family history, genetic testing may help in the identification. The initial use of clinical presentation and a family history of conductive deafness in the clinic requires further evaluation. In the long term, the literature is inconsistent with regard to surgical outcomes, but the results of this study suggest that stapedectomy may be successful and can lead to lasting improvements in hearing. This family showed autosomal dominant inheritance related to the NOG gene, which is significant for understanding the pathogenic characteristics and clinical features of stapes ankylosis syndrome.

\section{Abbreviations}

NOG:Noggin,

EDTA: Ethylene Diamine Tetraacetic Acid

SYM1: Symphalangism 1

SYNS1: Synostosis syndrome 1

ROM : Range of motion 


\section{Declarations}

\section{Acknowledgments}

The authors would like to thank the Vice Deanship of Scientific Research

Chair, Glaucoma Research Chair in Ophthalmology, King Saud University. We

also thank our clinical coordinator Mr. Abdulrahman Al-Mosa for his assistance

in the work.

\section{Authors' contributions}

$\mathrm{R}$ Y: Experimental design, analysis, data interpretations, wrote and edited the manuscript; $\mathrm{H}$ J: sample preparation, genotyping and data acquisition, manuscript editing and revision; W L:experimental design, recruitment, clinical diagnosis, surgery, data interpretation, manuscript editing and revision. All authors read and approved the final manuscript.

\section{Funding}

This work was supported by the Jiangxi Youth Science Foundation Project (20171BAB215034) and Subject of Jiangxi health and Family Planning Commission(2019B129,20183063)

\section{Availability of data and materials}

The datasets used and/or analysed during the current study are available from the corresponding author on reasonable request.

\section{Ethics approval and consent to participate}

The study adhered to the tenets of the Declaration of Helsinki and had received approval from the Institutional Review Board and Research Ethics Committee of The First Affiliated Hospital of Nan Chang University, Nanchang, Jiangxi (approval number 34352). Written, informed consent was obtained from all participants before their inclusion in this study.

\section{Consent for publication}

Written informed consent for publication of identifying images or other personal or clinical details was obtained from all of the participants.

\section{Competing interests}

The authors declare that they have no competing interests.

\section{Acknowledgements}




\section{References}

1.Shu $Y$, Wang $L$, Cheng $X$, et al. The p.(Pro170Leu) variant in NOG impairs noggin secretion and causes autosomal dominant congenital conductive hearing loss due to stapes ankylosis. J Genet Genomics. 2019;46(9):445-449.

2.Brown DJ, Kim TB, Petty EM, Downs CA, Martin DM, Strouse PJ, Moroi SE, Milunsky JM, Lesperance MM.. Autosomal dominant stapes ankylosis with broad thumbs and toes, hyperopia, and skeletal anomalies is caused by heterozygous nonsense and frameshift mutations in NOG, the gene encoding noggin. Am J Hum Genet. 2002;71(3):618-624.

3.Usami S, Abe S, Nishio S, Sakurai Y, Kojima H, Tono T, Suzuki N. Mutations in the NOG gene are commonly found in congenital stapes ankylosis with symphalangism, but not in otosclerosis. Clin Genet. 2012 Dec;82(6):514-520.

4.Ishino T, Takeno S, Hirakawa K. Novel NOG mutation in Japanese patients with stapes ankylosis with broad thumbs and toes. Eur J Med Genet. 2015;58(9):427-432.

5. Gong Y, Krakow D, Marcelino J, Wilkin D, Chitayat D, Babul-Hirji R, Hudgins L, Cremers CW, Cremers FP, Brunner HG,Reinker K, Rimoin DL, Cohn DH, Goodman FR, Reardon W, Patton M, Francomano CA, Warman ML (1999) Heterozygous Mutations in the gene encoding noggin affect human joint morphogenesis. Nat Genet 21:302-304

6. Liu F, Huang Y, Liu L, et al. Identification of a novel NOG mutation in a Chinese family with proximal symphalangism. Clin Chim Acta. 2014;429:129-133.

7.Mangino M, Flex E, Digilio MC, Giannotti A, Dallapiccola B. Identification of a novel NOG gene mutation (P35S) in an Italian family with symphalangism. Hum Mut at. 2002;19(3):308.

8.Ma C,Liu L,Wang FN,et al. Identification of a novel mutation of NOG in family wi th proximal symphalangism and early genetic counseling.BMC Med Genet.2019;20(1):169.

9.Takahashi T, Takahashi I, Komatsu M, et al. Mutations of the NOG gene in indiv iduals with proximal symphalangism and multiple synostosis syndrome. Clin Genet. 2001;60(6):447-451.

10.Declau F, Van den Ende J, Baten E, Mattelaer P. Stapes ankylosis in a family with a novel NOG mutation: otologic features of the facioaudiosymphalangism syndrome e. Otol Neurotol. 2005;26(5):934940 .

11.Hwang $\mathrm{CH}$, Wu DK. Noggin heterozygous mice: an animal model for congenital conductive hearing loss in humans. Hum Mol Genet. 2008;17(6):844-853. 
12. Milunsky J,Suntra C,MacDonald CB.Congenital stapes ankylosis,broad thumbs,a nd hyperopia:report of a family and refinement of a syndrome.Am J Med Genet.1999;82(5):404-408.

13. Teunissen B, Cremers WR. An autosomal dominant inherited syndrome with con genital stapes ankylosis. Laryngoscope. 1990;100(4):380-384.

14. Hilhorst-Hofstee Y, Watkin PM, Hall CM, Baraitser M. The autosomal dominant syndrome with congenital stapes ankylosis, broad thumbs and hyperopia. Clin Dysm orphol. 1997;6(3):195-203.

15. Bayat A, Fijalkowski I, Andersen T,et al. Further delineation of facioaudiosymph alangism syndrome: Description of a family with a novel NOG mutation and without hearing loss. Am J Med Genet A. 2016;170(6):1479-1484.

16. Takahashi T, Takahashi I, Komatsu M, et al. Mutations of the NOG gene in individuals with proximal symphalangism and multiple synostosis syndrome. Clin Genet. 2001;60(6):447-451.

17.Thomeer HG, Admiraal RJ, Hoefsloot L, Kunst HP, Cremers CW. Proximal symphalangism, hyperopia, conductive hearing impairment, and the NOG gene: 2 new mutations. Otol Neurotol. 2011;32(4):632-638.

18.Debeer P,Fryns JP, Devriendt K,et al.A novel NOG mutation Pro37 Arg in a family with tarsal and carpal synostoses.Am J Med Genet A,2004,128A(4):430-440.

19.Takano K, Ogasawara N, Matsunaga T, et al. A novel nonsense mutation in the NOG gene causes familial NOG-related symphalangism spectrum disorder. Hum Genome Var. 2016;3:16023.

20. Liu F, Huang Y, Liu L, et al. Identification of a novel NOG mutation in a Chinese family with proximal symphalangism. Clin Chim Acta. 2014;429:129-133.

21.Athanasakis E, Biarnés $X$, Bonati MT, Gasparini P, Faletra F. Identification of a New Mutation (L46P) in the Human NOG Gene in an Italian Patient with Symphala ngism Syndrome. Mol Syndromol. 2012;3(1):21-24.

22.Ganaha A, Kaname T, Akazawa Y, et al. Identification of two novel mutations in the NOG gene associated with congenital stapes ankylosis and symphalangism. J Hum Genet. 2015;60(1):27-34.

23.Pang X, Wang Z, Chai Y, et al. A Novel Missense Mutation of NOG Interferes With the Dimerization of NOG and Causes Proximal Symphalangism Syndrome in a Chinese Family. Ann Otol Rhinol Laryngol. 2015;124(9):745-751.

24.Vesell ES. Symphalangism, strabismus and hearing loss in mother and daughter. N Engl J Med. 1960;263:839-842.

25.Takahashi T,Takahashi I,Komatsu M,et al.Muations of the NOG gene in individu als with proximal symphalangism and multiple synostosis syndrome.Clin Genet,2001,60(6):447-451.

26. Y. Gong, et al. Heterozygous mutations in the gene encoding noggin affect hum an joint morphogenesis, Nat. Genet. 21 (3) (1999) 302-304. 
27. Ensink RJ,Sleeckx JP,Cremers CW. Proximal symphalangism and congenital conductive hearing loss:otologic aspects.Am J Otol.1999;20(3):344-349.

28. Weekamp HH, Kremer H, Hoefsloot LH, et al. Teunissen-Cremers syndrome: a clinical, surgical, and genetic report. Otol Neurotol. 2005;26(1):38-51.

29. Dixon ME, Armstrong P, Stevens DB, et al. Identical mutations in NOG can cause either tarsal/carpal coalition syndrome or proximal symphalangism. Genet Med. 200 1;3(5):349-353.

30. Lehmann K, Seemann P, Silan F, et al. A new subtype of brachydactyly type B caused by point mutations in the bone morphogenetic protein antagonist NOGG IN. Am J Hum Genet. 2007;81(2):388396.

\section{Figures}

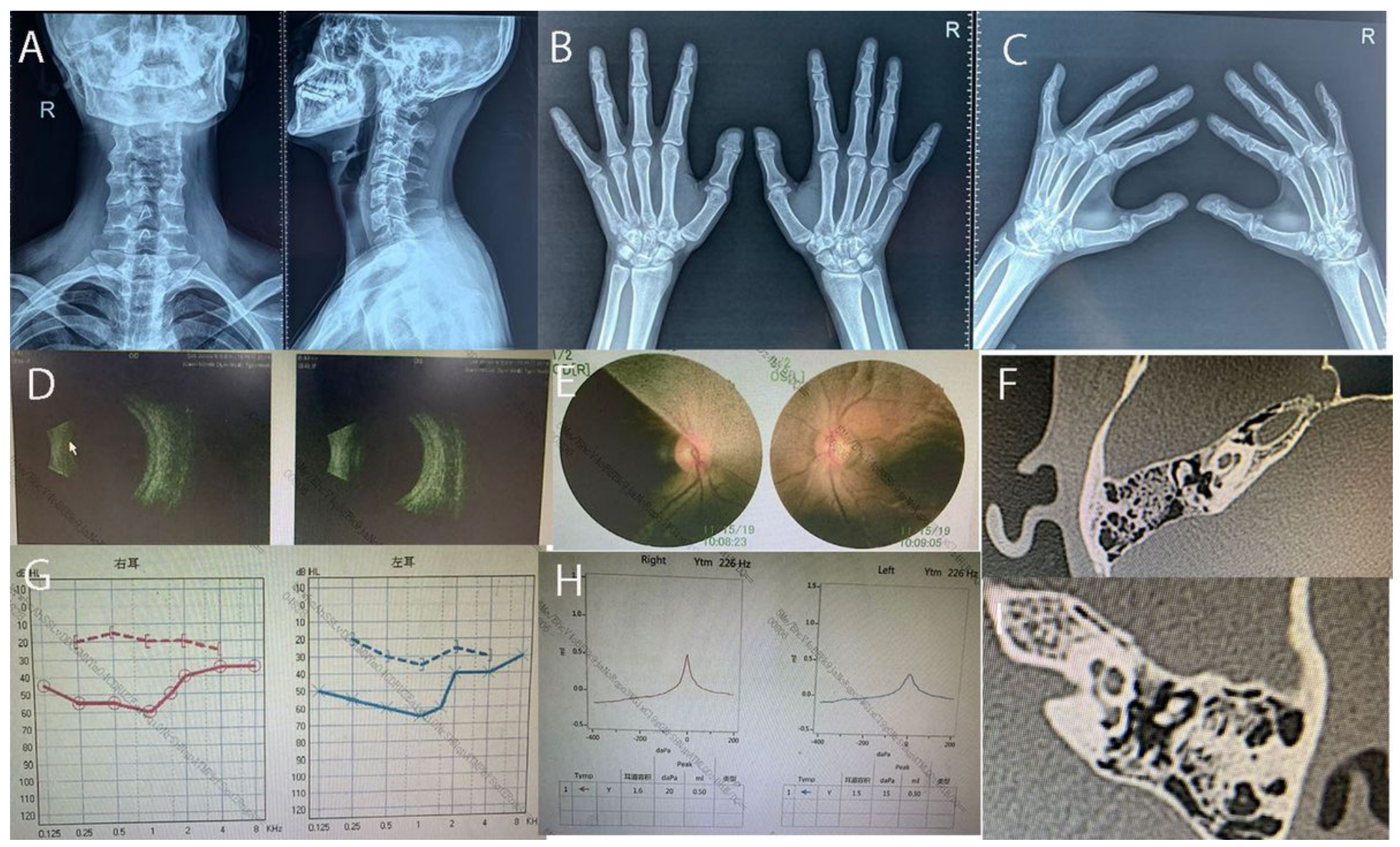

\section{Figure 1}

The audiological and physical examination of the probands (A) Cervical vertebra degener ation by X-ray

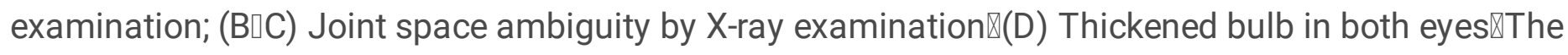
vitreous body of both eyes is punctate turbid by ultrasonography(E) no obvious abnormality by fundus camera $(\mathrm{G})$ Binaural conductive hearing by PTA; $(\mathrm{H})$ "A"type by acoustic immittance measurement; (FII) the temporal bone $\mathrm{CT}$; 


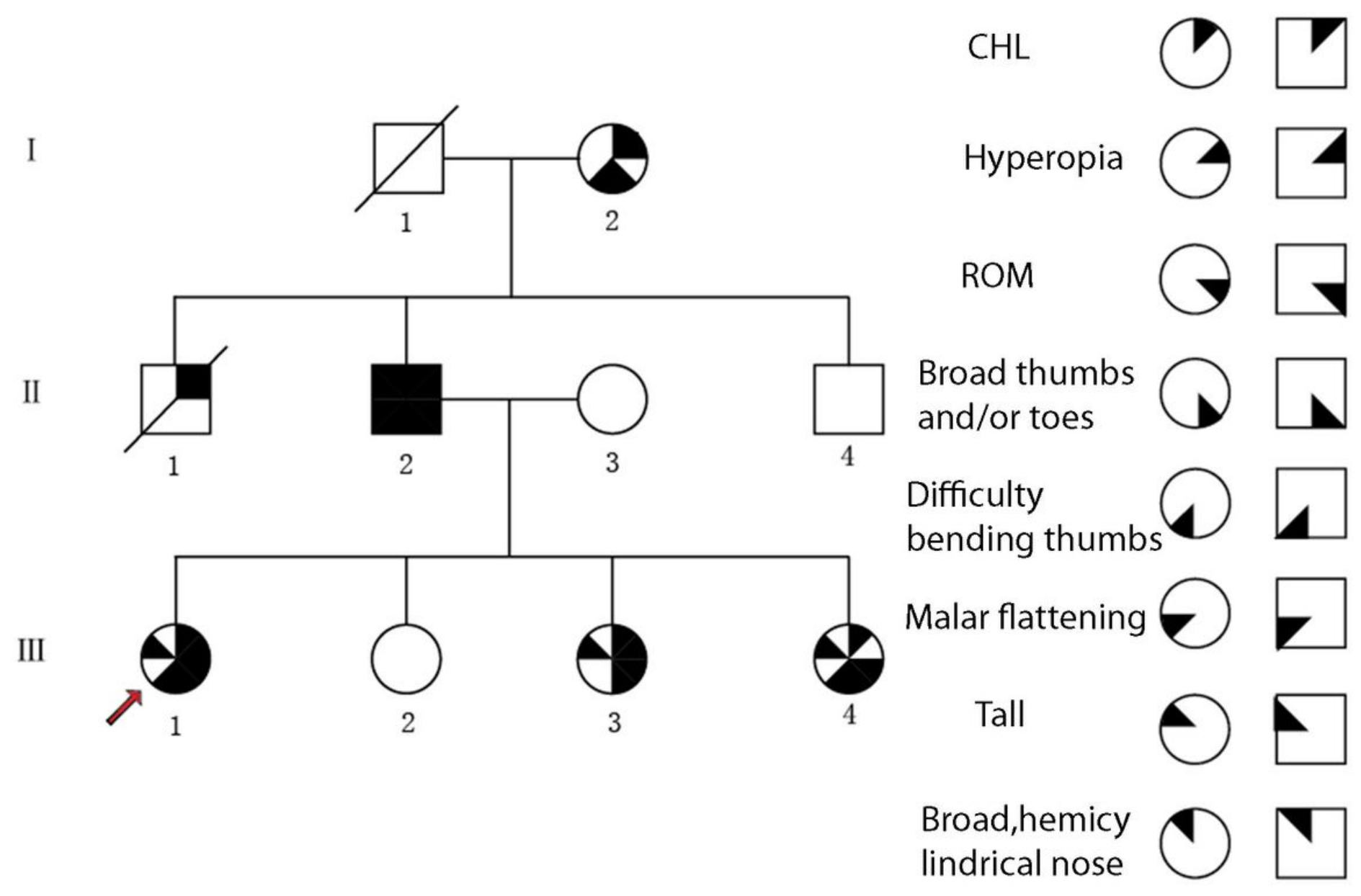

Figure 2

Pedigree of family. The arrow denotes the proband. Square symbols denote male patients, and circles denote female patients; blackened symbols denote affected individuals, and unblackene d symbols denote unaffected individuals; each quadrant defines a phenotypic element or a set of phenotypic elements,and blackened quadrants indicate the presence of the corresponding phenoty pic element(s) 


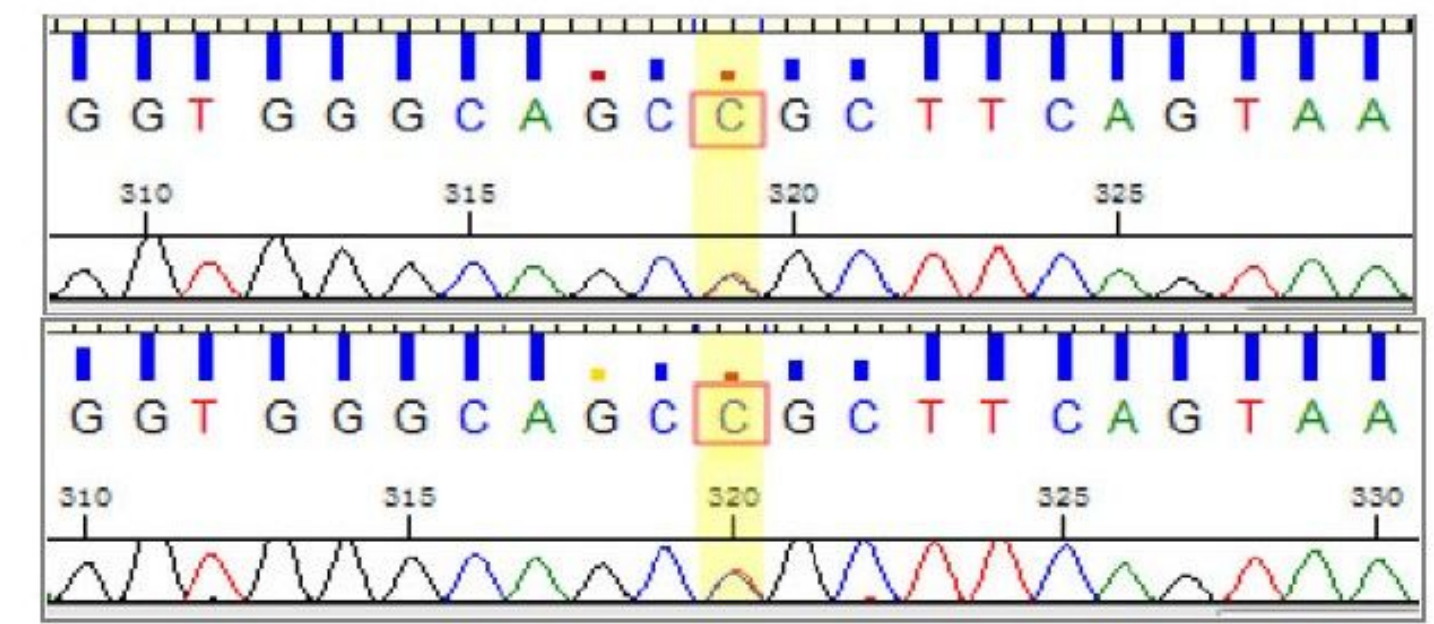

Father of
proband

Proband

B

C

Grandma of

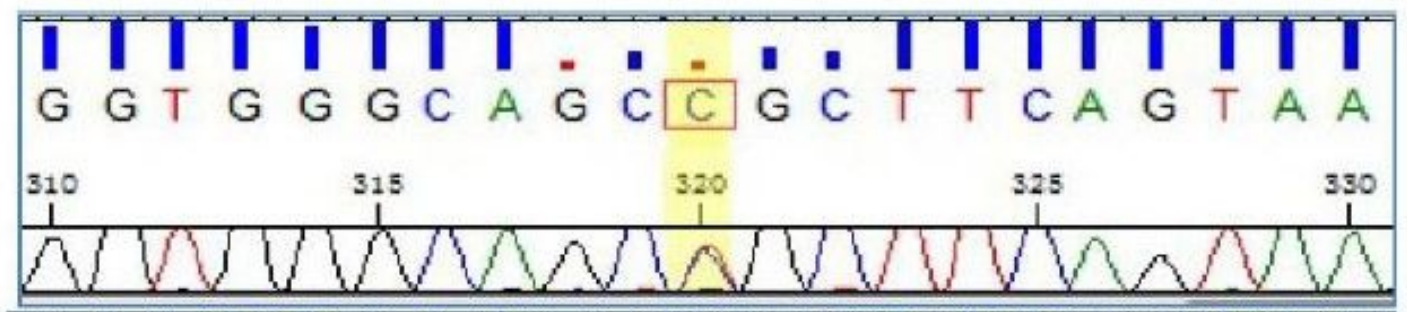

Elder sister

D

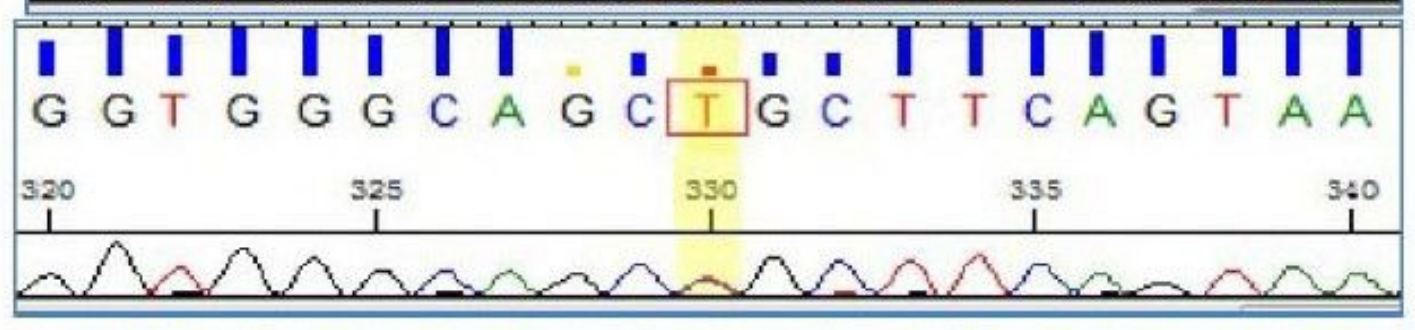

$E$

Younger sister

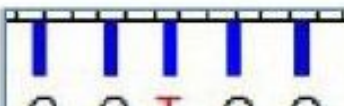

$G$ G T G G

of proband

310

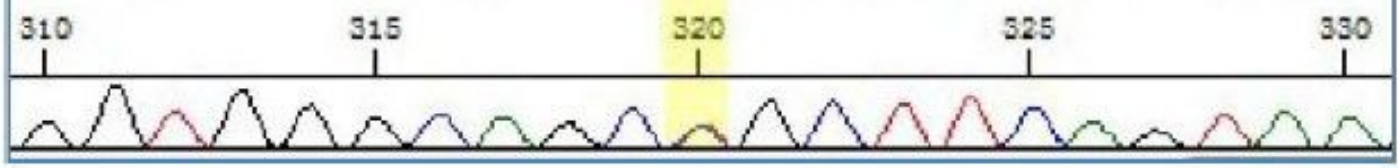

\section{Figure 3}

NOG c.532T>C(chr17:54672116) (A)Sequencing results of NOG gene individual total exon detection of the proband; (B)Sequencing results of NOG gene individual total exon detection of father of the proband(C)Sequencing results of NOG gene individual total exon detection of grandma of proband(D) Sequencing results of NOG gene individual total exon detection of father of elder sister of proband (E) Sequencing results of NOG gene individual total exon detection of father of younger sister of proband 